\title{
Impact of Medicare Part D on Medicare-Medicaid Dual-Eligible Beneficiaries' Prescription Utilization and Expenditures
}

\author{
Anirban Basu, Wesley Yin, and G. Caleb Alexander
}

Objective. To examine the effect of Part D on 65-78-year-old noninstitutionalized dual eligibles' prescription utilization and expenditures.

Data Source. Random sample of unique pharmacy customers of a national retail pharmacy chain who filled at least one prescription during both 2005 and 2006. For each subject, we obtained claims data for every prescription filled between January 1 , 2005, and April 31, 2007.

Study Design. Generalized estimating equations were used to examine the experience of a "treatment" group (dual eligibles between 65 and 78 years on January 1, 2005) with that of a "control" group (near-elderly patients with Medicaid coverage between 60 and 63 years on January 1, 2005) during the first 18 months after Part D implementation.

Principal Findings. Expenditures for the treatment and control groups tracked each other closely in the pre-Part D period. Immediately following the implementation of Part D, expenditures for both groups decreased and then leveled off. There were no significant changes in trends in the dual eligibles' out-of-pocket expenditures, total monthly expenditures, pill-days, or total number of prescriptions due to Part D.

Conclusions. We find no evidence that Part D adversely affected pharmaceutical utilization or out-of-pocket expenditures of dual eligibles during the transition period, nor during the 16 months subsequent to Part D implementation.

Key Words. Medicare Part D, dual eligibles, prescription utilization, out-of-pocket costs

The Part D drug benefit represents the single largest change to Medicare in its 40-year history. Because of the size of the program - the program enrolls over 25 million seniors and is expected to cost over U.S. $\$ 700$ billion in its first 10 years of implementation-considerable attention has been paid to studying the effect of the Part D benefit on aggregate drug utilization among seniors, seniors' out-of-pocket expenditures, total federal expenditures, and revenues 
of drug manufacturers (Bach and McClellan 2006; Lichtenberg and Sun 2007; Frank and Newhouse 2008; Yin et al. 2008), although this research has been hindered due to the sequestering of Medicare Part D claims data by the Center for Medicare and Medicaid Services (Stuart 2008).

There are, however, important reasons to examine the effect of the Part D drug benefit on specific subpopulations, especially the more than 6 million beneficiaries who were dually eligible for Medicare and Medicaid (dual eligibles). With Part D implementation, dual-eligible beneficiaries faced new rules governing their access to pharmaceuticals and were no longer subject to the same protections found under Medicaid law (Crowley, Ashner, and Elam 2005). ${ }^{1}$ Further, while dual eligibles pay no premium and face no deductible under Part D, they may face slightly higher copayments for covered drugs, or full out-of-pocket cost of drugs not included in their Part D plan formulary, although many states provide wrap-around coverage to these drugs statutorily excluded from Part D such as the benzodiazepines, but not all drugs. Potential adverse effects of instances of higher cost sharing are compounded by the relatively low income and asset levels among dual beneficiaries, and by random auto-enrollment of the vast majority of duals beneficiaries into Part $\mathrm{D}$ plans whose formularies may not include drugs demanded by specific individuals (Levinson 2006). In addition, dual-eligible beneficiaries have a higher incidence of disabling and chronic illness and lower incomes and education than the average Medicare population (Moon and Shin 2006), making these beneficiaries more vulnerable to coverage lapses due to the administrative complexity associated with transitioning from Medicaid to Part D.

Early data on dual eligibles' access to prescription drugs provided cause for concern. For example, in a telephone survey of employed dual eligibles in Kansas conducted in early 2006, 20 percent of participants reported difficulties obtaining medications, 13 percent were required to switch medications, and 8 percent stopped taking at least one medication (Hall, Kurth, and Moore 2007). In another study of a nationally random sample survey of psychiatrists, more than one-half (53 percent) of psychiatrists interviewed about the experiences

Address correspondence to Anirban Basu, Ph.D., 5841 S. Maryland Ave. MC-5000, Chicago, IL 60637; e-mail: abasu@medicine.bsd.uchicago.edu. Anirban Basu, Ph.D., and G. Caleb Alexander, M.D., are with the Section of General Internal Medicine, Department of Medicine, and Center for Health and the Social Sciences, University of Chicago, IL. Anirban Basu, Ph.D., is also with the National Bureau of Economic Research, Cambridge, MA. Wesley Yin, Ph.D., is with the Department of Economics, Boston University, Boston, MA. 
of a randomly selected dual-eligible patient reported that the patient had experienced medication access problem. More than one-fifth (23 percent) of these psychiatrists reported having discontinued or temporarily stopped a patients' medication because of prescription drug coverage or management issues (West, Wilk, and Muszynski 2007). Neuman et al. (2007) conducted a national survey in 2006 and found that dual-eligible seniors had the highest rates of changing Part D plans (11 percent) across all Part D enrollees. Some of these problems may have been due to system-level difficulties (such as identifying the low-income status of patients) in transition where the dual eligibles were incorrectly charged, improperly asked to pay deductibles, or not listed in plans they thought they were enrolled in.

These new rules and other challenges to implementing Part D were widely anticipated and there was considerable apprehension regarding the potential for disrupted access to prescription drugs for the dual eligibles (GAO 2005). In response, varying state contingency plans were developed to help transition dual eligibles into Part D plans. For example, during early 2006, 37 states implemented temporary coverage programs to provide low-income Medicare beneficiaries access to drugs through Medicaid (West, Wilk, and Muszynski 2007). Additionally, the Centers for Medicare and Medicaid Services required all Part D plans to have "transition plans," for example, to offer a one-time supply of drugs not included in the formulary but which were previously consumed by new enrollees (Smith et al. 2006). These programs may have played an important role in mitigating any short-run difficulties faced by dual eligibles during their transition between Medicaid and Part D coverage. For example, in a recent study (Madden et al. 2008), researchers have found evidence for a small but significant overall decrease in cost-related medication nonadherance following Part D implementation, but no such decrease among the sickest beneficiaries.

Only very recently, some analyses on the effect of Part D on the burden faced by dual eligibles are beginning to emerge. Shrank et al. (2008) have looked at the impact of Part D on dual eligibles' utilizations of five specific drugs and found no effect in any of them. To date, no empirical analyses have been performed to look at the effect of Part $\mathrm{D}$ on the overall burden faced by dual eligibles in terms of drug usage, out-of-pocket costs, and total drug expenditures. We attempt to fill this gap by using pharmacy claims from a national pharmacy chain accounting for approximately 15 percent of the outpatient prescription drug market in the United States. Therefore, based on a broad national sample of Medicare beneficiaries, we study how Part D impacted the drug utilizations and expenditures of this vulnerable population 
compared with their potential utilizations and expenditures had they continued under contemporaneous state Medicaid policies.

\section{METHODS}

\section{Data}

We selected a 5 percent random sample of unique pharmacy customers who filled at least one prescription both in the 2005 and in the 2006 calendar years at any retail or mail order member of a national pharmacy chain. This national pharmacy chain had presence in 45 states during 2005-2006, although about 40-50 percent of their sales came from five states: Florida, Texas, California, Illinois, and Arizona. Overall, this chain accounted for 15 percent of all retail prescription sales in United States. For each of these subjects, we obtained claims data for every prescription filled between January 1, 2005, and April 31, 2007. We formed two groups-a "treatment" group comprising of dual eligibles who were between 65 and 78 years old on January 1, 2005, and a "control" group comprising near-elderly patients with Medicaid coverage (and no Medicare coverage) between 60 and 63 years as of January 1, 2005. Within this pharmacy chain, this control group population is expected to age in the coming years to become the Part D eligible cohort that will be similar to our treatment group. Importantly, these near-elderly control subjects were not eligible for Medicare throughout our study period, thereby removing subjects who were dually eligible. ${ }^{3}$ For identification of Medicaid subjects, we looked for the use of at least one prescription that was reimbursed by Medicaid during the entire pre-Part D period of January 1, 2005, to December 31, 2005. Subjects identified as covered by Medicaid and ages 65-78 years as of January 1 , 2005 , constituted our sample of dual eligibles. Because of the infrequent churning of the dual eligibles in and out of Medicaid (Stuart and Singhal 2006), we relied on a single Medicaid prescription to identify dual eligibles. Using the Panel 9 of the Medical Expenditure Survey data, we estimated that in this age group, 85 percent remained continuously eligible for Medicaid between 2004 and 2005. Subjects in the control group were identified by their having filled at least one prescription reimbursed by Medicaid during both the pre- and postPart $\mathrm{D}$ periods. In order to ensure that we did not include nonelderly dualeligible patients in the control group, we excluded patients in this age range if any of their prescriptions were reimbursed by Medicare during the entire study period. Again using the Panel 9 of the Medical Expenditure Survey data, 
we estimated that in the 60-63 years age group, 88 percent remained continuously eligible for Medicaid between 2004 and 2005.

For each prescription claim, we obtained data including subjects' demographic characteristics (age, sex, language preference, zip code of residence), insurance characteristics (prescription drug plan, method of payment), pharmacy characteristics (zip code location), prescription characteristics (National Drug Code [NDC], therapeutic class, drug dose, number of treatment days, date dispensed, number of refills), and expenditures (amount paid out of pocket, amount paid by third party). We used data on subjects' zip code of residence (i.e., the residence recorded at subjects' first pharmacy claim in 2005) to link the pharmacy claims data to data from the 2000 Census, including information on the total population, median household income, income per capita, fraction urban, fraction African American, unemployment rate, and poverty rate within the zip code of residence.

\section{Statistical Analyses}

We studied the effect of Medicare Part D on four pharmaceutical outcomes using regression discontinuity designs: (1) total number of prescriptions per month, (2) pill-day-a prescription utilization measure similar to medication possession ratio that counts the number of days with a pill summed across all prescriptions, (3) monthly out-of-pocket costs, and (4) total prescription expenditures. Each subject in our sample contributed 28 observations corresponding to 28 months of data.

We divided the 28 months into three periods. The first period, January 2005 to December 2005, represented the "pre-Part D" period. The second period, January 2006 to May 2006, represented months during which various state-level and federal-level temporary measures, installed to facilitate transition, are believed to have influenced utilization and is referred to as the "transition post-Part D" period (Fox and Schofield 2006; Health and Human Services Commission Report 2007). The third period, June 2006 to April 2007, represented months following this initial transition period and is called the "stable post-Part D period."

To estimate the impact of Part D, we estimated the trends and breaks in the trends at the beginning of each period for each of the outcome variables among the dually eligible sample and compared them with a counterfactual trend that would have been present had there been no Part D. To estimate counterfactual trends, we used the contemporaneous time profile of the corresponding outcomes in a control group of Medicaid-eligible subjects aged 
60-63 years. We first confirmed that the trends in outcomes in the 60-63-yearold cohort provided an adequate control for the subjects in our elderly dualeligible group during the pre-Part D era (September 2004 to December 2005). To do so, we examined both the statistical significance and the substantive relevance of the coefficients in our regression model that represented the differential trends between the dual-eligible and the control group during the pre-Part D era. We then used the predicted control trends in the post-Part D era to represent the outcomes in the dual-eligible group had there been no Part $\mathrm{D}$ policy in place.

These trends were estimated and predictions were generated using generalized estimating equations (GEE). We adjusted for subject demographics (gender, English-speaking status, Medicaid coverage, and age), zip-code level characteristics (total population, median household income, per-capita income, urbanicity, proportion black, unemployment rate, and poverty), fixed effects for calendar months (January-December), periods (pre-Part D, transition post-Part D, and stable post-Part D), and the treatment group. ${ }^{4}$ We used a gamma distribution for modeling out-of-pocket and total expenditures and negative binomial distributions for modeling pill-days and number of prescriptions. All estimators employed log-link models. Additionally, we explored the correlation structure between monthly observations within subjects and finally employed a first-order auto-regressive correlation structure for out-of-pocket costs and number of prescriptions and an unstructured correlation matrix for pill-days. In our fully specified model, we estimated the impact of Part D during both the transition period and "stable" period after the May 31, 2006, and allowed for both the intercept (break in trend) and the slope of outcome variable trend lines to differ. Details about the specifications of these models are given in Appendix SA2.

Note that for the near elderly to be a good control for dual-eligible subjects, secular trends in their prescription drug expenditures and utilization must be similar. We tested whether trends in outcomes among the 60-63-yearold sample were similar to our dual-eligible sample group during the pre-Part D period (January 2005 to December 2005). To do so, we tested whether the slopes of the trend lines during the pre-Part $\mathrm{D}$ period differed significantly. We expected there to be a difference in mean level of each outcome variable between the two groups; however, the difference in the outcome variables needed to be constant over the pre-Part $\mathrm{D}$ period in order for the near elderly to suffice as a good control.

Next, we examined continuation and discontinuation, as some of the heterogeneity in medication switches may be masked in the total utilization 
measures that we studied above. ${ }^{5}$ Using multinomial regressions, we estimated the probability of continuing, discontinuing, or initiating a medication with a specific NDC code between the pre-Part D period and the stable Part D period. We studied the differential rate of continuation between dual eligibles and the control group. In an adjoining analysis, we restricted our analysis to only those NDC codes that were filled in the pre-Part D period. Using logistic regression, we estimated the probabilities of continuation as a function of the number of prescription filled in the pre-Part D period. Higher number of prescriptions filled in the preperiod indicates a chronic use of that medication that patients should be more likely to continue in the next period.

Finally, we used the same difference-in-difference strategy discussed earlier to examine changes in the overall percentage of generic prescription use between pre-Part D and stable post-Part D period and contrasted them between the dual-eligible and control group patients with at least one prescription in the stable postperiod. Standard errors for all parameters were obtained via 1,000 bootstrap replicates clustered by subjects, and a variety of model fit criteria was used to check the goodness of fit for our models. ${ }^{6}$ All analyses were performed with Stata version 9.2 (Stata Corp., College Station, TX).

\section{RESULTS}

Table 1 describes basic characteristics of our dual-eligible $(n=10,837)$ and control cohorts $(n=3,199)$. Dual eligibles were older and fewer preferred English as their primary language, but they were otherwise comparable to the control group of patients. Despite being younger, our control group had greater average annual drug utilization and expenditures, which may reflect both the severity of health conditions for elderly Medicaid patients about to enter Medicare and the death of the more severely ill patient before reaching older ages (mortality effects). Unadjusted changes between pre- and post-Part $\mathrm{D}$ periods were not significant for any of the drug utilization measures for either the control or the dual-eligible group.

\section{Trends in Expenditures and Utilization}

Figure 1A shows the trends in average monthly out-of-pocket expenditures for dual eligibles and the control group of near-elderly subjects ages 60-63. Expenditures for the two groups tracked each other closely in the pre-Part D period, suggesting that the near elderly sufficed as a comparison group. Trends in expenditures continued to track each other closely in the post-Part D period. 
Table 1: Description of Subjects Filling Prescriptions within National Pharmacy Chain

\begin{tabular}{|c|c|c|c|c|}
\hline & \multicolumn{2}{|c|}{$\begin{array}{c}\text { Control Subjects on } \\
\text { Medicaid 60-63 Years Old } \\
(n=3,199)\end{array}$} & \multicolumn{2}{|c|}{$\begin{array}{c}\text { Treatment Dual } \\
\text { Eligibles 66-79 Years Old } \\
(n=10,837)\end{array}$} \\
\hline Age (years) (SD) & \multicolumn{2}{|c|}{$61.8(1.1)$} & \multicolumn{2}{|c|}{$72.2(3.7)$} \\
\hline Female sex $(\%)$ & \multicolumn{2}{|c|}{67.3} & \multicolumn{2}{|c|}{69.4} \\
\hline English language preference (\%) (SD) & \multicolumn{2}{|l|}{92.1} & \multicolumn{2}{|c|}{82.3} \\
\hline \multicolumn{5}{|c|}{ Characteristics of zip code based on 2000 Census } \\
\hline Total population (thousands) (SD) & \multicolumn{2}{|c|}{$29.6(16.9)$} & \multicolumn{2}{|c|}{$32.1(17.5)$} \\
\hline $\begin{array}{l}\text { Median household income } \\
\text { (thousands) (SD) }\end{array}$ & \multicolumn{2}{|c|}{$19.2(6.6)$} & \multicolumn{2}{|c|}{$20.8(8.2)$} \\
\hline Income per capita (thousands) (SD) & \multicolumn{2}{|c|}{$7.9(3.0)$} & \multicolumn{2}{|c|}{$8.5(3.9)$} \\
\hline Fraction urban population $(\%)$ & \multicolumn{2}{|l|}{91.8} & \multicolumn{2}{|c|}{94.3} \\
\hline Fraction African American (\%) & \multicolumn{2}{|l|}{22.2} & \multicolumn{2}{|c|}{19.2} \\
\hline Unemployment rate $(\%)$ & \multicolumn{2}{|l|}{92.1} & \multicolumn{2}{|c|}{92.4} \\
\hline \multirow[t]{2}{*}{ Poverty rate $(\%)$} & \multicolumn{2}{|l|}{16.7} & \multicolumn{2}{|c|}{15.9} \\
\hline & Pre-Part D & Post-Part D & Pre-Part D & Post-Part D \\
\hline $\begin{array}{l}\text { Total monthly prescription drug } \\
\text { utilization, pill-days (SD) }\end{array}$ & $103(128)$ & $106(132)$ & $96(115)$ & $99(121)$ \\
\hline $\begin{array}{l}\text { Total monthly number of } \\
\text { prescriptions (SD) }\end{array}$ & $3.6(4.4)$ & $3.6(4.4)$ & $3.1(3.6)$ & $3.1(3.7)$ \\
\hline $\begin{array}{l}\text { Total monthly out-of-pocket prescription } \\
\text { expenditures, U.S.\$ (SD) }\end{array}$ & $13.8(65.8)$ & $14.1(66.1)$ & $14.9(65.4)$ & $14.7(65.1)$ \\
\hline $\begin{array}{l}\text { Total monthly prescription } \\
\text { expenditures, U.S.\$ (SD) }\end{array}$ & $222(367)$ & 227 (390) & $178(275)$ & $183(304)$ \\
\hline
\end{tabular}

Notes. The sample of 177,311 subjects includes 59,663 unique control group subjects (ages 60-63), and 117,648 treatment group subjects (ages 66-79), observed in both 2005 and 2006. Standard deviations $(\mathrm{SD})$ are reported in parentheses.

In fact, the joint test of the three coefficients that represent the interactions of the cubic monthly splines with the dual-eligible group and the pre-Part D era (Appendix SA2) was not significant $(p$ value $=.11)$. Immediately following the implementation of Part D, expenditures for both groups continued to decrease during the transition period and then leveled off.

Figure 1B compares the trend in actual observed expenditures to the trend in predicted expenditures assuming Part D was never implemented (this is a "counterfactual" trend calculated using parameter estimates from the GEE regression analysis). ${ }^{4}$ There were no significant changes in trends in the dual eligibles' out-of-pocket expenditures due to Part D. Similar results were found for total monthly expenditures (Figure 1C and D); pill-days (Figure 2A and B); and total number of prescriptions (Figure $2 \mathrm{C}$ and $\mathrm{D}$ ). The formal results are 
Figure 1: Observed and Predicted Time Trends in Outcomes for Dual Eligibles and the Control Group
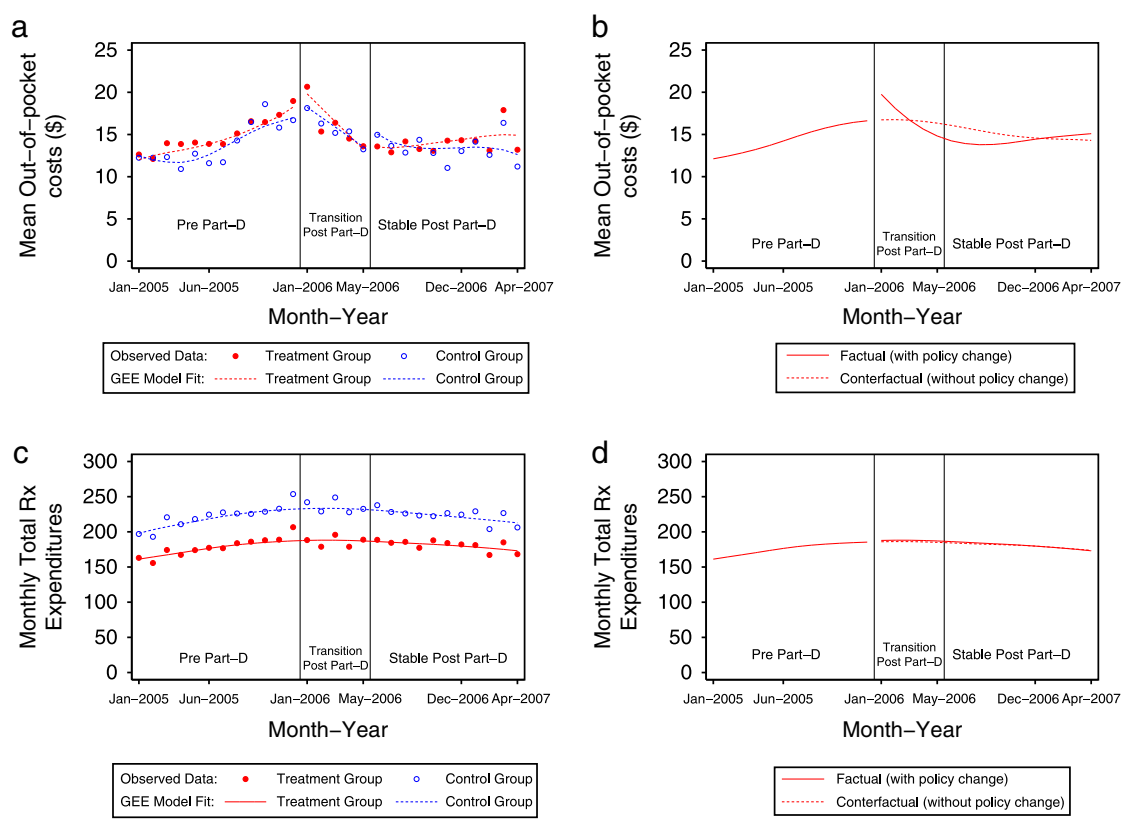

The corresponding factual and counterfactual trends post-Part D for the dual eligibles are also presented.

summarized in Table 2. Although slightly higher levels of changes were found for each of the outcomes in the transition period than the stable period, these changes were neither meaningfully large in magnitude nor statistically different from zero.

\section{Probability of Starting, Stopping, or Continuing Medication}

Next, we studied the probability of continuing, discontinuing, or initiating a new medication between pre-Part $\mathrm{D}$ and the stable phase of post-Part $\mathrm{D}$. We found that among all medications for which at least one prescription was filled in either of the two periods, dual eligibles filled a prescription for 17.5 percent of the medications in both the pre- and postperiods (continuation), filled a prescription in the preperiod but not in the postperiod for 42.5 percent of the medications (discontinuation), and filled a prescription in the postperiod but not in the preperiod for 40 percent of the medication (initiation) (Table 3). 
Figure 2: Observed and Predicted Time Trends in Outcomes for Dual Eligibles and the Control Group
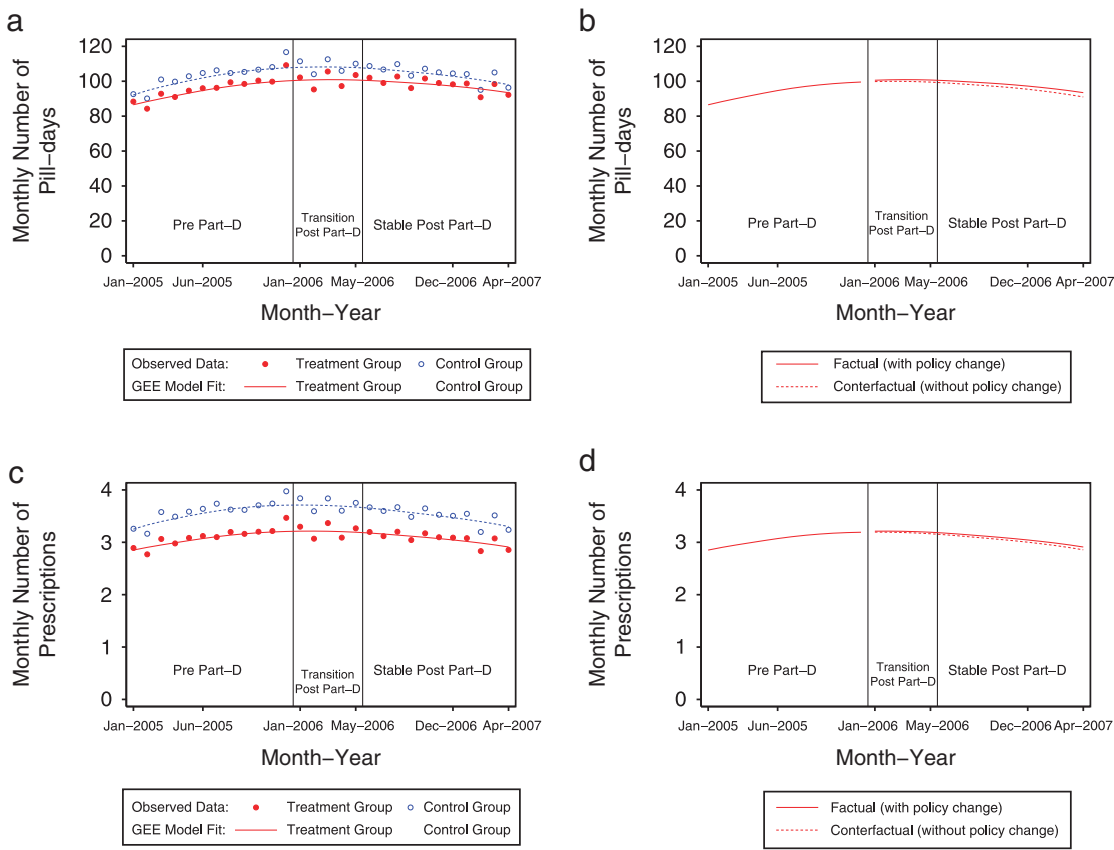

The corresponding factual and counterfactual trends post-Part D for the dual eligibles are also presented.

These proportions were almost identical for the control group patients, implying that Part D did not meaningfully impact patterns of prescription usage among dual eligibles.

Focusing on only those medications that were used in the pre-Part D period, we found that the probability of filling a prescription for the same medication following Part $\mathrm{D}$ increased with the number of prescriptions filled in the preperiod among both beneficiaries and control subjects. This supports the fact that patients who used a medication in a chronic manner were more likely to continue to use it over time. However, as before, we did not find any difference in the probability of continuation between dual eligibles and the control group at any level of preperiod usage (Figure 3).

Finally we studied the proportion of generic prescriptions used between the pre- and the post-Part D period. We found that compared with 
Table 2: Impact of Part D on Out-of-Pocket Prescription Expenditures and Prescription Utilization

\begin{tabular}{|c|c|c|c|c|}
\hline & \multicolumn{2}{|c|}{$\begin{array}{l}\text { Average-Adjusted Monthly Outcomes } \\
\text { for Beneficiaries Ages 66-79 }\end{array}$} & \multicolumn{2}{|c|}{ Differences Due to Part D } \\
\hline & Actual Outcomes & $\begin{array}{l}\text { Predicted Outcomes } \\
\text { without Part D }\end{array}$ & Absolute Change & Percent Chang \\
\hline \multicolumn{5}{|l|}{ Transition period } \\
\hline OOP Rx costs (U.S.\$) & $16.5(0.48)^{+}$ & $17.8(1.33)^{+}$ & $-1.25(1.19)$ & $-7.0(6.7)$ \\
\hline Total Rx costs (U.S.\$) & $186.1(2.7)^{+}$ & $180.7(5.4)^{+}$ & $5.49(4.9)$ & $3.0(2.7)$ \\
\hline Pill-days & $100.7(0.91)^{+}$ & $99.1(1.59)^{+}$ & $1.60(1.24)$ & $1.6(1.2)$ \\
\hline Total no. of Rx & $3.21(0.03)^{+}$ & $3.18(0.05)^{+}$ & $0.03(0.04)$ & $0.9(1.25)$ \\
\hline \multicolumn{5}{|l|}{ Stable period } \\
\hline OOP Rx costs (U.S.\$) & $14.2(0.49)^{+}$ & $14.7(2.12)^{+}$ & $-0.50(2.19)$ & $-3.2(14.9)$ \\
\hline Total $\mathrm{Rx}$ costs (U.S.\$) & $181.2(2.9)^{+}$ & $181.6(7.2)^{+}$ & $-0.43(7.2)$ & $-0.2(3.9)$ \\
\hline Pill-days & $98.0(0.91)^{+}$ & $96.3(2.02)^{+}$ & $1.61(1.66)$ & $1.7(1.7)$ \\
\hline Total no. of $\mathrm{Rx}$ & $3.07(0.03)^{+}$ & $3.03(0.06)^{+}$ & $0.04(0.06)$ & $1.3(2.0)$ \\
\hline
\end{tabular}

Notes. Counterfactual average monthly outcomes calculated as from predicted values of the GEE models; overall effects reported in the third column are calculated as differences between actual and counterfactual outcomes; overall outcomes as a percentage of the predicted outcomes without Part D are reported in the fourth column.

Impact reported as average monthly expenditures or utilization and reported separately for period before Part D enrollment deadline ("transition period") and period following Part D enrollment deadline ("stable period").

${ }^{+} p<.05$; standard errors obtained via 500 bootstrapped replicates.

the preperiod, dual eligibles were 4.8 percent more likely to use generic prescriptions in the postperiod (Table 3). However, we see a similar change in the control group too, which suggests that this change was not due to Part D.

Table 3: Adjusted Probabilities for Discontinuing, Continuing, and Initiating New Medications in Post-Part D Stable Period Compared with Pre-Part D Period in the Dual Eligibles and the Control Group

\begin{tabular}{lccr}
\hline & Dual Eligibles & Control & Difference \\
\hline Probability of & & & \\
Discontinuing & 42.5 & 43.6 & -1.1 \\
Continuing & 17.5 & 18.1 & -0.6 \\
$\quad$ Initiating & 40.0 & 38.3 & 1.7 \\
\% point change in & 4.8 & 4.6 & 0.2 \\
Generics Rx use* & & 4.6 & \\
\hline
\end{tabular}

Note. $p<.05$; standard errors obtained via 500 bootstrapped replicates.

*Among patients with at least $1 \mathrm{Rx}$ in the stable post-Part $\mathrm{D}$ period. 
Figure 3: The Average Probability of Continuing a Medication (NDC-CodeSpecific), Filled in the Pre-Part D Era, in the Post-Part D Stable Period

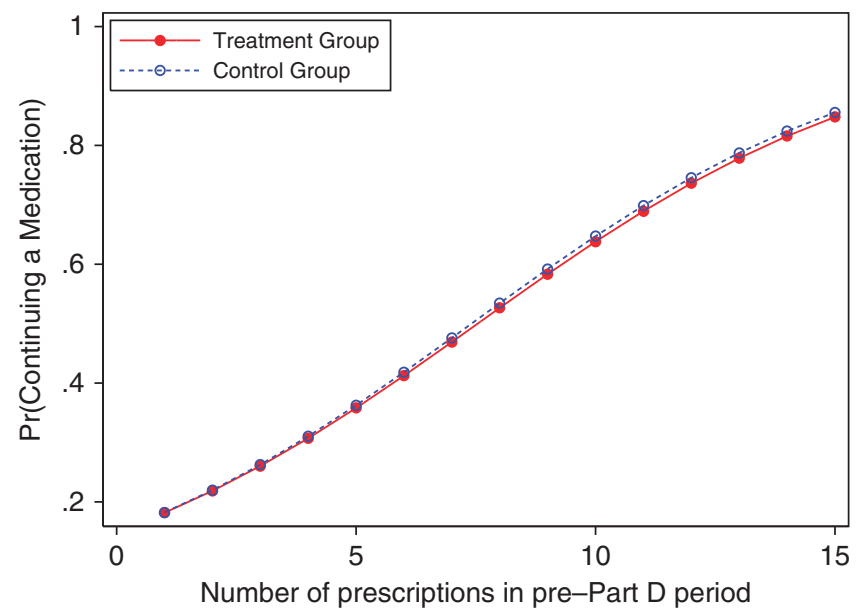

\section{DISCUSSION}

Despite designing Part D benefits for dual eligibles to protect this vulnerable population against the lack of continuity of necessary drugs, several concerns regarding its implementation and its promise of seamless access to drugs were raised at the time of transition. In this analysis of a diverse sample of dual eligibles accounting for a substantial portion of the overall U.S. market of prescription drugs, we found that Part D did not adversely impact either prescription utilization or expenditures among beneficiaries dually eligible for Medicare and Medicaid. In addition, our results suggest that other aspects of dual eligibles' prescription utilization, such as the initiation of new therapies and the rates of generic drug utilization, were also not adversely impacted by Part D. Our results conform to the results from other analyses that have looked at the effect of Part D on long-term utilization trends among dually eligible patients (Shrank et al. 2008). Our results are also in contrast to other results (Hall, Kurth, and Moore 2007; West, Wilk, and Muszynski 2007) that have looked at specific subpopulations of dually eligible patient and found diminished access to drugs immediately following Part D implementation. One of the limitations of these previous analyses is that a short-term assessment does not do justice to the long-term impact of Part D among the dual eligibles, 
since they were permitted to change Part $\mathrm{D}$ plans monthly and possibly select the more optimal plan over time. ${ }^{7}$ Moreover, what is lacking in many of these analyses is a relevant control group.

Our findings of no adverse impact of Part $\mathrm{D}$ on dual eligibles both in the period immediately following and in the 16 months post-Part $\mathrm{D}$ implementation are important to mitigate the previously anticipated concerns and also to highlight the joint role of federal and state policy makers who devoted substantial efforts to ensuring that the transition to Part D would not lead to unintended consequences among this group. Perhaps, the most important of these efforts were the rapid steps taken by several state governments in allocating additional funds to cover drugs for dual eligibles during the first 3 months of Part D implementation, during which pharmacies, patients, and providers figured out the nuances of the disintegrated system of PDPs and the varying formularies that accompanied them. Our results are not driven by any idiosyncratic policy changes, such as prior authorizations, within this pharmacy chain.

Our findings hinge on the assumption that trends in drug utilizations and expenditures among dual eligibles would have continued similarly to those of our control group had Part D not been implemented. To that extent, we found that our control group, which consisted of near-elderly Medicaid patients, showed a peak in utilization at the end of 2005 followed by a modest decline in 2006, which could be driven by several reasons: preferred drug lists (PDLs) were implemented by many State Medicaid agencies throughout 2005 and 2006 (in fact, all states implemented at least one cost containment strategy in FY 2006), and these PDLs and also prior authorization requirements were made stringent in many states in 2006 as they were preparing to participate in drug purchasing pools (Health and Human Services Commission Report 2007). More importantly, the Deficit Reduction Act of 2005 was enacted on February 8, 2006, under which states had been given more authority to control Medicaid drug spending through increased cost sharing for nonpreferred drugs, changes in the way Medicaid pays pharmacists, allowing pharmacists to refuse prescriptions for beneficiaries who do not pay their cost sharing, and inclusion of authorized generic drugs in the calculation of "best price" for drugs (Kaiser Commission on Medicaid and the Uninsured 2006). These ongoing changes in Medicaid may have contributed to the peak in utilization for the control group at the end of 2005. However, further evidence confirming this conjecture is required. In our analysis, we expect that had Part $\mathrm{D}$ not been introduced, dual eligibles would also have been subjected to these Medicaid policies. Therefore, the post-Part D trends of the control group represent a 
reasonable counterfactual for the post-Part D dual eligibles had they continued under Medicaid policies.

We also found, using national expenditure datasets, that prescription expenditures and utilizations grew until 2005 and then dropped in 2006 among near-elderly Medicaid patients. We looked at this in two ways. First, we estimated the national $\mathrm{Rx}$ monthly expenditures and the monthly average number of Rx among 55-63 year olds under Medicaid who did not have Medicare during 2003, 2004, 2005, and 2006 using MEPS data. They were estimated to be U.S.\$198, U.S.\$201, U.S.\$218, and U.S.\$207, respectively, and 3.13, 3.25, 3.47, and 3.39, respectively. Second, we looked at the same Medicaid cohort over time (more in line with our data), that is, 53-61 in 2003, 54-62 in 2004, 5563 in 2005, and 56-64 in 2006, and found their national Rx monthly expenditures to be U.S.\$198, U.S.\$202, U.S.\$218, and U.S.\$206, respectively. Their monthly average numbers of $\mathrm{Rx}$ were $3.09,3.39$, 3.47, and 3.33, respectively. The modest national spikes in expenditure and utilizations among middleaged Medicaid patients in 2005 conform to the notion of anticipation behavior due to the stringent implementation of PDLs as a result of states participating in drug-purchasing pools and implementing stricter cost-sharing rules. ${ }^{8}$

Although our data are not nationally representative and may not characterize the experience of all dual eligibles, the data offer an important opportunity to examine the impact of this marked change in prescription coverage for millions of Americans. Furthermore, in prior analyses, our subjects were found to be similar to a nationally representative sample (Yin et al. 2008), and estimates of the overall impact of Part D on prescription utilization were highly consistent with those predicted by economic rationales (Pauly 2004). However, the experiences for the dual-eligible patients that we find in our study may also be attributed to resources allocated by this pharmacy chain, which may not be representative of the national experiences of all dual eligibles.

There are other limitations of our study. One limitation of our study is that we assume that the absence of a prescription claim for an individual subject represents zero utilization for that subject, rather than missing data. However, individuals may obtain their prescriptions from more than one pharmacy chain, and thus it is possible that subjects observed here obtained only part of their medicines from this chain alone. Our previous analysis (Yin et al. 2008) of a subsample of subjects for whom we have complete prescription benefits manager data suggested that similar large proportions of subjects of each age group ( $>90$ percent) filled all of their prescriptions within the pharmacy chain in both 2005 and 2006, and we applied inclusion criteria 
requiring subjects to have at least one prescription claim during both 2005 and 2006. Although loyalty to one pharmacy chain would not threaten our conclusions (due to our use of a similarly defined control group), any correlation between loyalty and Part D enrollment would influence findings drawn from this serial cross-sectional analysis. For example, restrictions on state-level pharmacy networks by Part D plans might induce some of the enrollees to move to other pharmacies. In such a case, we would expect to see downwardly biased estimates for utilizations under Part D from one pharmacy chain, which will make our policy effect estimates liberal (bigger than they actually are). A higher rate of switching among Part D group, due to restricted coverage, in itself may represent disruption of service, even though by not capturing this aspect of the data, we produce liberal (upwardly biased) estimates of the effect of Part D on the levels of Rx use. However, given that we find a null effect even with an upwardly biased estimator leads us to believe that differential switching rate is not a very influential driver of disruption in this case.

A second limitation of our study, which is also general to most pharmacy claims data, is that we are unable to observe drug utilizations for individuals during periods of hospitalization during our study period. However, we do not think that this is a serious limitation of our results, as unless the difference in rates and length of hospitalizations between pre- and post-Part $\mathrm{D}$ periods are differentially changing for dual eligibles and the control group, our differencein-difference estimator should be able to account for most of these biases. Finally, we do not observe eligibility criteria directly but only infer about them indirectly through utilization. Consequently, our method of identifying the control group may have included some dual eligibles who do not incur any Medicare utilization during our observation period, although the fraction of such people may be quite small in these data.

\section{CONCLUSIONS}

Part D represents a policy change of enormous proportions. Any undertaking as complex as this is bound to face challenges, and particularly during the transition period during the first few months of the benefit, there was considerable concern about the impact of the transition on dual eligibles. Some of the previous research on this topic that had tried to capture these experiences of the dual-eligible patients during the Part $\mathrm{D}$ transition period seems to indicate that this policy had adversely affected their drug access and utilization. However, our report suggests that Part D had no effect on dual-eligible pa- 
tients' prescription utilization and expenditures over a longer time frame compared with their potential utilization and expenditures had they continued under contemporaneous state Medicaid policies. Our results are in line with other reports that have also looked at long-term outcomes. However, as Part D plans keep churning and exiting this market, dual eligibles are also being moved across plans continuously. Moreover, due to the heterogeneity in Medicaid coverage of prescription drugs across states, substantial state-level variation in the impact of Part $\mathrm{D}$ may prevail. Therefore, monitoring the degree of access and health outcomes for this group seems to continue to be an important topic within this dynamic policy area.

\section{ACKNOWLEDGMENTS}

Joint Acknowledgment/Disclosure Statement: We thank the editors and two anonymous reviewers for their comments that have helped enrich the discussions in the paper and present our methods more clearly. We also thank Atonu Rabbani for excellent research assistance. All errors are ours.

Disclosures: None.

Disclaimers: None.

\section{NOTES}

1. For example, some dual eligibles faced changes in cost sharing as many of the prevailing Medicaid plans did not charge copayments for drugs, while under Part $\mathrm{D}$, dual eligibles face fixed copayments for generic and brand therapies. Moreover, dual-eligible individuals also faced new or more stringent formularies and formulary management tools enforced by the privately administered prescription drug plans (PDPs). In theory, choice among PDPs available within a Medicare region would allow dual eligibles to select the plan with the formulary that best matches their needs. However, duals who failed to exercise such selection were randomly enrolled into a standard PDP that may have negatively impacted prescription utilization and cost-sharing relative to preexisting Medicaid coverage (see Crowley, Ashner, and Elam 2005).

2. The precise age cut-off for the control group was 62 years and 8 months as of January 1, 2005, so that they do not become eligible for Medicare at the end of our analysis period. Also, we exclude 64 and 65 year olds as of January 1, 2006, since they had partial year Medicare eligibility and would transition into the dual-eligible status in the middle of our time frame of analysis. 
3. However, a majority of Medicaid-only recipients in that age group are likely to be disabled and eligible for Medicaid due to SSI eligibility. We thank one of the anonymous reviewers for pointing this out.

4. We explored a variety of splines for representing differential time trends between periods. Misspecification of the degree of spline within any period led to mis-fitted models that in turn generated biased results. Ultimately, we used cubic splines because they produced the best fit for both the pre-Part D and the stable post-Part $\mathrm{D}$ periods. A linear spline was used for the transition post-Part $\mathrm{D}$ period due to the short time interval in this period. We added up to three-way interactions between the period and treatment group fixed effects and the splines over time. We assessed overall model fit using a variety of goodness-of-test criteria.

5. Our goal in examining "continuity" was not to focus clinically on medication adherence or appropriateness of therapies but rather to conduct a population-level analysis of the impact of Part D on overall utilization. Although analyses of broad therapeutic categories may be clinically meaningful, such efforts may not suffice to describe the overall policy effect since patients with multiple comorbidities (such as those in this population) may have to substitute across therapeutic classes due to lack of access. Therefore, we aggregated medicines from all therapeutic classes when examining "continuity, discontinuity, and initiation" of therapies. To address the concern that some of these medications were not intended to be continued, we use our difference in difference estimator to recover the impact of Part D.

6. Model fit criteria to check the goodness of fit for our models included the Pearson correlation test between residuals and predicted values, Hosmer Lemeshow test examining systematic biases in predictions across deciles of the corresponding linear predictors, and Pregibon link test examining residual nonlinearity in outcomes with respect to covariates. Although all of these tests were statistically significant for both the outcomes, none showed any substantive bias in predictions. For example, the Pearson correlations coefficient for out-of-pocket costs and pilldays were -0.009 and 0.004 , respectively, and yet they were statistically significant ( $p$-values $<.001$ for both). The overall model fits are illustrated in Figures 1A, $\mathrm{C}$ and $2 \mathrm{~A}, \mathrm{C}$ and appear to track the trends in observed outcome well.

7. While evidence on this is only beginning to emerge, theoretically one might expect a greater churning among dual eligibles for two reasons. First, they were involuntarily assigned a PDP at the start of the Part D program and so many individuals may not have been matched to a program that was optimal for their needs. Second, unlike regular Part D enrollees who are allowed to switch plans once a year, dualeligible beneficiaries are allowed to switch to a different PDP at any time with coverage under a new PDP effective the following month. More importantly, dual eligibles who changed plans reflect a select group who were facing challenges with their old plans. Therefore, even if churning is small, the overall impact may be large due to the self-selected nature of churning. Recent evidence presented at the 2009 Academy Health Meeting (Bishop et al. 2009) seem to suggest that about 20\% of dual eligibles switched their auto-assigned plans by the 2-year mark.

8. Evidence of such anticipatory prescribing behavior or "stockpiling" can be found in the literature (Marshall et al. 2002; Wang et al. 2008). 


\section{REFERENCES}

Bach, P. B., and M. B. McClellan. 2006. "The First Months of the Prescription-Drug Benefit-A CMS Update." New England Journal of Medicine 354: 2312-4.

Bishop, C., C. P. Thomas, D. Gilden, and J. Kubisiak. 2009. Enrollment of Dually Eligible Beneficiaries in Medicare Part D Plans: Autoassignment and Choice. Academy Health Annual Research Meeting (Abstract), Chicago, IL.

Crowley, J., D. Ashner, and L. Elam. 2005. State Medicaid Outpatient Prescription Drug Policies: Findings from a National Survey, 2005 Update [accessed on June 1, 2009]. Menlo Park, CA: The Kaiser Commission on Medicaid and the Uninsured. Available at http://www.kff.org/medicaid/7381.cfm

Fox, K., and L. Schofield. 2006. The Pharmacy Coverage Safety Net: Variations in State Responses to Supplement Medicare Part D. Mimeo, University of Southern Maine.

Frank, R. G., and J. P. Newhouse. 2008. "Should Drug Prices Be Negotiated under Part D of Medicare? And If So, How?" Health Affairs 27 (1): 33-43.

General Accountability Office (GAO). 2005. Contingency Plans to Address Potential Problems with the Transition of Dual-Eligible Beneficiaries from Medicaid to Medicare Drug Coverage. GAO-06-278R, transmitted from GAO to Senate Finance Committee on December 16, 2005, by letter from Kathleen M. King, Director, Health Care, GAO, to Senator Max Baucus.

Hall, J. P., N. K. Kurth, and J. M. Moore. 2007. "Transition to Medicare Part D: An Early Snapshot of Barriers Experienced by Younger Dual Eligibles with Disabilities." American Journal of Managed Care 13 (1): 14-8.

Health and Human Services Commission Report. 2007. "Analysis of Multi-State Medicaid Drug Purchasing Pool” [accessed on March 10, 2009]. Available at http:/www.hhsc.state.tx.us/reports/Multi-State_MedicaidDrugPurchasing.pdf

Kaiser Commission on Medicaid and the Uninsured. 2006. "Deficit Reduction Act of 200: Implications for Medicaid" [accessed on March 10, 2009]. Available at http://www.kff.org/medicaid/7465.cfm

Levinson, D. R. 2006. "Dual Eligibles' Transition: Part D Formularies' Inclusion of Commonly used Drugs." Inspector General's Office, Department of Health and Human Services [accessed on March 2008]. Available at http://www.oig.hhs. gov/oei/reports/oei-05-06-00090.pdf

Lichtenberg, F., and S. Sun. 2007. "The Impact of Medicare Part D on Prescription Drug Use by the Elderly: Evidence from a Large Retail Pharmacy Chain.” Health Affairs 26: 1735-44.

Madden, J. M., A. J. Graves, F. Zhang, A. S. Adams, B. A. Briesacher, D. Ross-Degnan, J. H. Gurwitz, M. Pierre-Jacques, D. G. Safran, G. S. Adler, and S. B. Soumerai. 2008. "Cost-Related Medication Nonadherance and Spending on Basic Needs Following Implementation of Medicare Part D." Journal of the American Medical Association 299 (16): 1922-8.

Marshall, J. K., P. V. Grootendorst, B. J. O'Brien, L. R. Dolovich, A. M. Holbrook, and A. R. Levy. 2002. "Impact of Reference-Based Pricing for Histamine-2 Receptor Antagonists and Restricted Access for Proton Pump Inhibitors in British Columbia." Canadian Medical Association Journal 166 (13): 1655-62. 
Moon, S., and J. Shin. 2006. "Health Care Utilization among Medicare-Medicaid Dual Eligibles: A Count Data Analysis." British Medical Journal Public Health 6: 88.

Neuman, P., M. K. Strollo, S. Guterman, W. H. Rogers, A. Li, A. M. C. Rodday, and D. G. Safran. 2007. "Medicare Prescription Drug Benefit Progress Report: Findings from a 2006 National Survey of Seniors." Health Affairs 26 (5): w630-43.

Pauly, M. V. 2004. "Medicare Drug Coverage and Moral Hazard." Health Affairs (Millwood) 23: 113-22.

Shrank, W. H., A. R. Patrick, A. Pedan, J. M. Polinski, L. Varasteh, R. Levin, N. Liu, and S. Schneeweiss. 2008. "The Effect of Transitioning to Medicare Part D Drug Coverage in Seniors Dually Eligible for Medicare and Medicaid." Journal of the American Geriatric Society 56 (12): 2304-10.

Smith, V., K. Gifford, S. Kramer, and L. Elam. 2006. The Transition of Dual Eligibles to Medicare Part D Prescription Drug Coverage: State Actions during Implementation. Results from a 50-State Snapshot.

Stuart, B. 2008. "Where Are the Medicare Part D Claims Data?" Annals of Internal Medicine 148: 1-2.

Stuart, B., and P. Singhal. 2006. The Stability of Medicaid Coverage for Low-Income Dually Eligible Medicare Beneficiaries. Menlo Park, CA: Kaiser Family Foundation Policy Brief. Publication \#7512.

Wang, P. S., A. R. Patrick, C. R. Dormuth, J. Avorn, M. Maclure, C. F. Canning, and S. Schneewiss. 2008. "The Impact of Cost Sharing on Antidepressant Use among Older Adults in British Columbia." Psychiatric Services 59: 377-83.

West, J. C., J. E. Wilk, I. L. Muszynski, D. S. Rae, M. Rubio-Stipec, C. L. Alter, W. E. Narrow, and D. A. Regier. 2007. "Medication Access and Continuity: The Experiences of Dual-Eligible Psychiatric Patients during the First 4 Months of the Medicare Prescription Drug Benefit." American Journal of Psychiatry 164 (5): 789-96.

Yin, W., A. Basu, J. Zhang, A. Rabbani, D. O. Meltzer, and G. C. Alexander. 2008. "Impact of the Medicare Part D Prescription Drug Benefit on Drug Utilization and Out-of-Pocket Expenditures." Annals of Internal Medicine 148: 169-77.

\section{SUPPORTING INFORMATION}

Additional supporting information may be found in the online version of this article:

Appendix SA1: Author Matrix.

Appendix SA2: Econometric Specifications.

Please note: Wiley-Blackwell is not responsible for the content or functionality of any supporting materials supplied by the authors. Any queries (other than missing material) should be directed to the corresponding author for the article. 